\title{
Automated evaluation of the surface point quality in dimensional X-ray computed tomography
}

\author{
Automatisierte Auswertung der Oberflächenpunktqualität bei dimensionellen \\ Röntgencomputertomografiemessungen
}

https://doi.org/10.1515/teme-2019-0116

Received August 23, 2019; accepted January 3, 2020

\begin{abstract}
X-ray computed tomography (CT) enables dimensional measurements of numerous measurands with a single scan, including the measurement of inner structures. However, measurement artefacts complicate the applicability of the technology in some cases. This paper presents a methodology to assess the surface point quality of computed tomography measurements without the requirement of a CAD model. Measurement artefacts lowering the surface point quality can therefore automatically be detected. The correlation of quality values with the random measurement error is demonstrated. The presented method can in principle be used to weight single fit points to reduce the measurement uncertainty of CT measurements.
\end{abstract}

Keywords: Industrial X-ray computed tomography, surface point quality, volume data, segmentation.

Zusammenfassung: Die Röntgencomputertomografie ermöglicht die Erfassung von vielen dimensionellen Messgrößen mit einem einzelnen Scan, wobei auch innenliegende Strukturen gemessen werden können. Dennoch schränken Messartefakte die Anwendung dieser Technologie in vielen Fällen ein. Dieser Artikel präsentiert eine Methode zur Beurteilung der Oberflächenpunktqualität von Röntgencomputertomografiemessungen, ohne dass hierfür ein CAD-Modell benötigt wird. Messartefakte, die Oberflächenqualität einschränken, können dadurch automatisch erkannt werden. Die Korrelation der Qualitätskennwerte mit den zufälligen Messabweichungen wird aufgezeigt. Die vorgestellte Methode kann prinzipiell dazu verwendet werden, Messpunkte bei der weiteren Auswertung einzeln zu gewichten und dadurch die Messunsicherheit

\footnotetext{
*Corresponding author: Andreas Michael Müller, Institute of Manufacturing Metrology (FMT), Friedrich-Alexander-Universität Erlangen-Nürnberg Erlangen, Germany, e-mail: andreas.mueller@fmt.fau.de Lorenz Butzhammer, Florian Wohlgemuth, Tino Hausotte, Institute of Manufacturing Metrology (FMT), Friedrich-Alexander-Universität Erlangen-Nürnberg Erlangen, Germany
}

von Röntgencomputertomografiemessungen zu reduzieren.

Schlagwörter: Industrielle Röntgencomputertomografie, Oberflächenpunktqualität, Volumendaten, Segmentierung.

\section{Introduction}

While CT has been used in the field of nondestructive testing for several decades, it is still a comparably new technology for dimensional metrology applications. Device improvements leading to higher dimensional accuracy fueled a fast growth of industrial application cases in the metrology field over the past years. There are three major advantages of CT in comparison with conventional coordinate measuring systems. First, inner or hidden geometry elements can be measured, which are not accessible for many other coordinate measuring systems. Second, the measurement time does not depend on the number of measurement features. Third, the work piece is completely captured and additional features can subsequently be evaluated based on the stored data, which makes CT attractive especially for complex parts.

Several effects leading to systematic measurement errors limit the applicability of CT for dimensional measurements. These measurement artefacts are often-times hard to detect. The surface point quality methodology presented in this paper allows the experienced user the automated detection and classification of measurement artefacts, if additionally information about the geometry of the measurement setup is taken into account. Consequently, this leads to a locally resolved assessment of the measurement quality of the work piece surface and allows for the identification of measurement artefacts as well as their reduction in some cases. Some examples of the capabilities of the approach are demonstrated. The basic principle of the underlying method was already published in 2015 [9] and [10] using the acronym "LQV" (Local Quality Value). Since then, the method has been expanded and signifi- 
cantly improved, especially regarding the efficiency of the data processing pipeline.

After a short introduction into the principle of CT and typical measurement artefacts, a description of the presented method is given, followed by several use cases in the context of dimensional metrology.

\section{Industrial X-ray computed tomography}

Industrial X-ray computed tomography is based on recording X-ray images of an object from different angles and reconstructing a volumetric representation of the object from these images. For dimensional metrology applications, an object surface is extracted from this volumetric image. A measurement setup consists of an X-ray source, an X-ray detector and a manipulator system as well as a computational evaluation unit (for reconstruction and evaluation). For the purpose of this paper, we restrict the discussion to industrial cone beam CT systems with conventional X-ray tubes. In these systems, an X-ray tube (typical X-ray energies up to $450 \mathrm{keV}$ [5]) emits X-rays towards a rectangular flat-panel detector. The measured object is placed between source and detector and is rotated by $360^{\circ}$ using a rotary stage. X-ray images of the object are measured at numerous angles (up to several thousand, depending on the setup) with constant angular increment.

The X-ray intensity reaching each detector pixel for a constant X-ray energy is described by the Beer-Lambert law [4]

$$
I=I_{0} \mathrm{e}^{-\int_{L} \mu(\vec{x}) \mathrm{d} \vec{x}}
$$

where $L$ indicates the line integral from the X-ray source to the detector pixel, $I_{0}$ denotes the intensity without attenuating object and $\mu(\vec{x})$ the local X-ray attenuation coefficient. For typically relevant X-ray energy ranges and materials, the main attenuation contributions are due to photoelectric absorption and Compton scatter [4] (see section 2.4). Real X-ray tubes emit X-rays of multiple energies, the artefacts caused by neglecting the polychromatic spectrum are discussed in section 2.2.

After image acquisition from all angles, a volume representation needs to be reconstructed from those detector images. For further processing, the projection integral for each pixel and rotation angle is used [4]:

$$
p=-\ln \frac{I}{I_{0}}=\int_{L} \mu(\vec{x}) \mathrm{d} \vec{x}
$$

The projection images created thereby are typically further processed using the fast and easy to implement FDK reconstruction algorithm [8], which is an approximative filtered backprojection approach for cone beam CT. The projections are weighted and row-wise high pass filtered to take the cone beam setup into account and to preserve high frequency information (e.g. edges). To avoid the enhancement of noise, the high pass filter is superimposed with a window function which handles the cut off at high frequencies. Different window functions are used in practice. The so called Shepp-Logan window [24] represents a popular one for industrial purposes, leading to a good compromise between sharp edges and noise enhancement. The backprojection process smears the filtered projection values from the detector pixels back along each ray through the volume.

While the FDK algorithm leads to exact results for the horizontal midplane, for every other point in space, missing data components can lead to cone-beam artefacts (see section 2.1).

CT reconstruction yields a three dimensional voxelised grey value volume. For performing dimensional measurements, the part surface needs to be determined from this voxel dataset (see chapter 4.3.3 of [5] for details). Ideally, the grey value profile perpendicular to a part surface should have the shape of a Heaviside function. The simple approach to divide interior and exterior based on their grey values are thus single isovalue thresholds - any voxel with a grey value above the isovalue is characterised as material, any other as air. The ISO50 value, the mean of the air- and material-peak grey values in the grey value histogram of the whole volume, is a widespread choice for the isovalue. For ideal monochromatic X-ray scans of homogeneous materials, showing no noise and no undersampling or discretisation artefacts, the ISO50 approach should yield accurate results. Surface determination on the subvoxel scale can be performed by interpolation of the grey values between the discrete voxel grid points. For real measurements, effects such as noise, polychromaticity (compare section 2.2), projection unsharpness and different penetration lengths lead to locally varying grey value levels in the measurement data such that systematic measurement errors are induced when using a constant isovalue threshold. Due to imperfections like projection and detector unsharpness, the grey value profile perpendicular to the surface is broadened into a smooth transition (compare Fig. 1) having a width correlating with the volumetric resolution (as e. g. measured by the modulation transfer function according to [1]). Therefore, isovalues determined locally or gradient approaches are commonly 


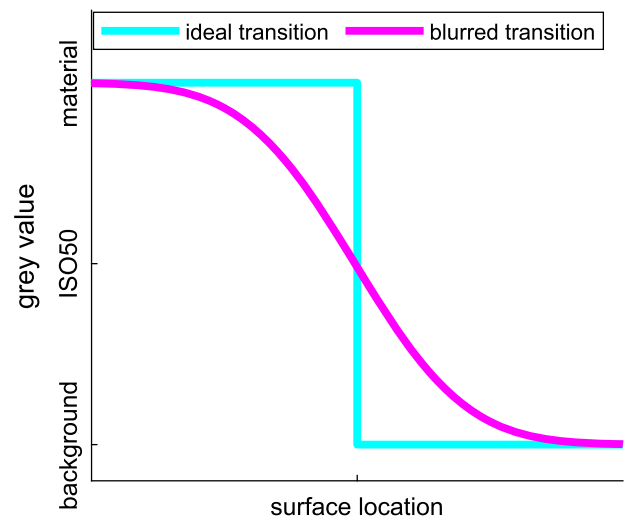

Figure 1: Idealised grey value profile in the volume normal to the part surface. The cyan line shows the ideal profile while the magenta line shows an idealised profile affected by image unsharpness (but e. g. free of noise).

used for surface determination. Gradient approaches locate the surface at the maximum norm of the grey value gradient [16].

The quality of surface determination is directly impacted by both the local absolute difference between material and air grey value as well as the gradient. Higher differences and gradients are expected to lead to better measurement precision [7]. In an ideal scan, the location of maximum gradient and the ISO50 threshold would coincide.

\subsection{Cone beam artefacts}

The $360^{\circ}$ rotation of the measurement object can be expressed as a circular trajectory of the X-ray source in the fixed coordinate system of the object. However, a circular trajectory is not satisfying the Tuy sufficiency condition [25], which requires that every plane through the measurement object is crossing the source trajectory at least once. It is therefore inherent to cone beam CT with a circular trajectory that there is data missing in the so called Radon space, which would be required for proper reconstruction. Bartolac et al. [3] give a good description for the causes and appearance of cone beam artefacts, which is especially useful regarding dimensional measurements where a proper reconstruction of material transitions (workpiece surface) is crucial for a high measurement accuracy. They show that nonmeasurable planes of the volume can be described by a shift-variant cone in the frequency domain (see Fig. 2).

If the normal vector of the local workpiece surface lies inside this cone, the frequency components of the material transition in this direction are not covered by the data acquisition process and the transition appears blurred after reconstruction. From Fig. 2 it can be seen that nonmeasurable planes are orientated nearly horizontally. The minimum tilt angle to avoid cone beam artefacts however is not constant but depends on the position in space. The opening angle of the cone increases with the distance to the trajectory plane. Therefore, cone beam artefacts become more prominent (see Fig. 3).

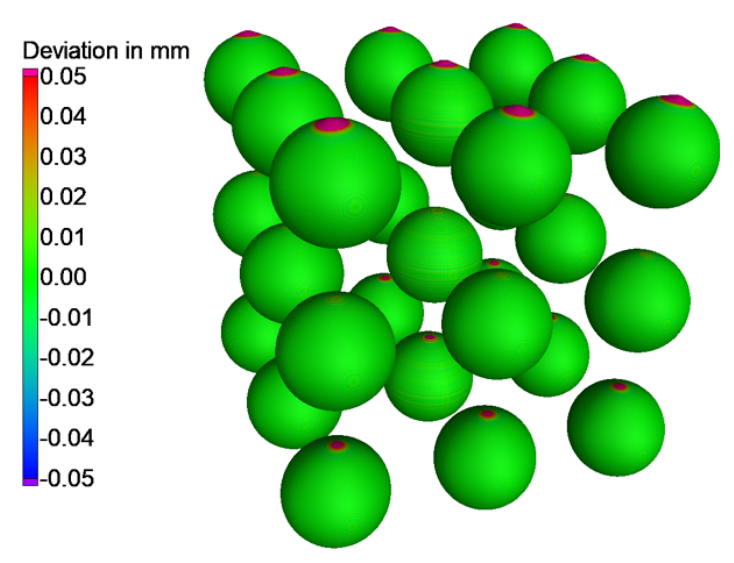

Figure 3: Target-actual comparison for a simulated CT measurement of a stack of spheres. The position dependency of the cone beam artefacts are clearly visible. Settings for simulation: Monochromatic point source, no scatter, noise or detector blur.
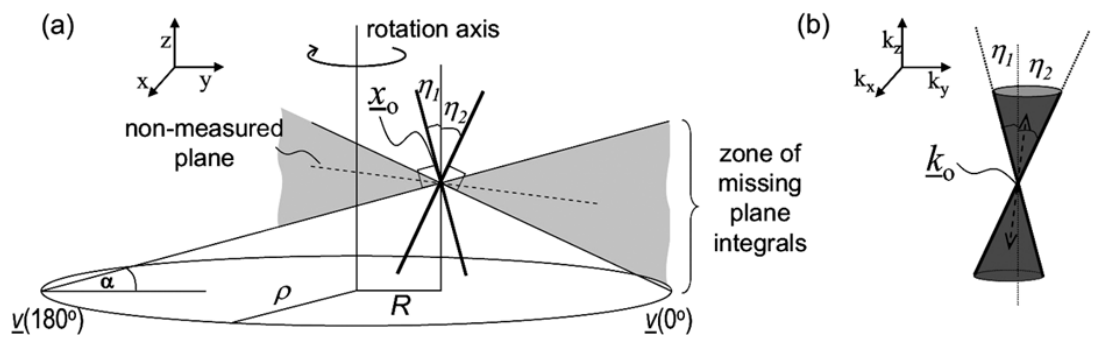

Figure 2: Representation of missing data in cone beam CT according to Bartolac et al. [3]. (a) Nonmeasurable planes for a point $x_{0}$ in space. (b) Representation of missing data in the frequency domain. Reprinted from [3]. 


\subsection{Beam hardening}

X-ray tubes which are used as beam source in industrial CT devices have a polychromatic spectrum, respectively energy distribution. Furthermore, the absorption coefficient $\mu$ depends on the photon energy. As for technically relevant cases, the absorption coefficient decreases with higher energy, the percentage of absorbed photons is higher for lower energies. The mean energy of the spectrum after absorption increases and the beam is "hardened".

To account for the polychromatic spectrum and the energy dependence of $\mu$, equation (1) has to be adapted to [26]

$$
I_{\text {poly }}=I_{0} \int_{0}^{E_{\max }} w(E) \mathrm{e}^{-\int_{L} \mu(\vec{x}, E) \mathrm{d} \vec{x}} \mathrm{~d} E,
$$

where $w(E)$ is a weighting function representing the source spectrum. The energy characteristics of the detector can also be included in the weighting function [15]. The projection integral for the polychromatic case accordingly has to be adapted to

$$
p_{\text {poly }}=-\ln \left(\int_{0}^{E_{\max }} w(E) \mathrm{e}^{-\int_{L} \mu(\vec{x}, E) \mathrm{d} \vec{x}} \mathrm{~d} E\right) .
$$

The reconstruction algorithm however assumes a linear behaviour, which can be expressed by [26]

$$
p=\int_{L} \int_{0}^{E_{\max }} w(E) \mu(\vec{x}, E) \mathrm{d} E \mathrm{~d} \vec{x} \neq p_{\text {poly }} .
$$

In the considered case, the measured projection integrals are smaller than expected. The deviations increase with higher penetration length and with higher variation of the absorption coefficient. As the projection integrals are underestimated more for inner regions of the workpiece with inherently high penetration lengths from all rotational orientations than for regions e. g. at the outer part surface, the grey values of these inner regions are lower after reconstruction. This phenomenom is called cupping artefact, which is well visible for example for upright scanned cylinders. Furthermore, the nonlinearity of equation (4) can lead to dark bands and streak artefacts between highly absorbing parts [2] and a reduced contrast for cavities. While the influence of cupping on dimensional measurements can be reduced by avoiding a global isovalue threshold for surface segmentation [15], streaks and local inhomogeneities can significantly increase measurement deviations.

\subsection{Photon starvation}

Severe artefacts in a CT measurement can arise if the material thickness or absorption is so high that the intensity decreases to the noise level of the detector without radiation. A further increase of absorption or penetration length can not be detected in this case, leading to an underestimation of the projection integral similarly as discussed in section 2.2 and to an amplification of noise [2]. Dark bands and streaks arise in the reconstructed volume. As the described effect is primarily visible for measurements of plastic parts with disturbing, highly absorbing metal inserts, the superimposed artefacts from beam hardening and photon starvation are often called metal artefacts (see e. g. [5]), following the terminology from medical CT.

\subsection{Scatter}

In the $\mathrm{X}$-ray energy regime below $1 \mathrm{MeV}$, the attenuation is caused by elastic Rayleigh scatter, photoelectric absorption and inelastic Compton scatter (see [4, 14] for details). Elastic scatter is usually negligible while the attenuation is dominated by photoelectric absorption and Compton scatter. Photoelectric absorption does usually only lead to negligible secondary radiation. Compton scatter is inelastic and thus there is a photon with a lower energy and a different propagation direction after scattering. This photon causes a signal not accounted for by equation (1) if it still reaches a detector pixel (which is not along its initial path). The scattering directions are not isotropic and a relevant percentage is scattered with a relatively small angle (especially at high incident photon energies [14]). Therefore, scattered photons cause a scatter intensity that the reconstruction model does not take into account. The linearity of equation (5) is thus again not given and artefacts arise similar to the case of beam hardening. Cupping, streaks and a loss of contrast can therefore also have their origin in scattered radiation [23].

\section{Evaluation of the surface point quality (SPQ)}

This chapter describes a method to evaluate the surface point quality (SPQ) of a dimensional CT measurement. The approach aims at calculating various quality parameters for discrete points on a determined surface. These parameters can then be used to compare surface regions with regard to their quality (e. g. caused by local CT-specific arte- 
facts) and subsequently provide the possibility of selective data processing during the evaluation of a measurement result. The main idea is to evaluate the CT volume data in form of the grey value transition in the proximity of the determined surface of the measurement object. This is the reason, why a profound understanding of industrial $\mathrm{X}$-ray CT including the propagation of error sources and influences through and onto the measurement chain is required to interpret the calculated SPQ. In order to apply the method, the following components need to be prepared:

- volume data of a CT measurement,

- determined surface with known coordinate transform with regard to the volume grid coordinate system,

- sufficient computing resources.

The demonstrated workflow is based on CT data processing using the software suite VGStudio Max (VGS), Volume Graphics GmbH, Heidelberg, Germany. For the calculation and evaluation of the surface point quality, a software prototype - SPQ evaluation program (SPQEP) - was developed at the Institute of Manufacturing Metrology (FMT) using the mathematical computing software MATLAB, The MathWorks, Natick, US-MA. Firstly, a CT measurement is performed and the corresponding reconstructed volume data is imported into VGS and a user defined surface determination is performed. The surface is then exported using the .stl file format, which defines surface data consisting of connected triangles, using the global "Scene" coordinate system of VGS. Ideally, the triangle mesh exhibits a homogeneous surface point distribution, having in mind that the computational requirements increase at least linearly with the number of surface points. Additionally, the affine transformation matrix describing the relation between the "Scene" coordinate system and the "Volume Grid" coordinate system is exported. This is required, because the base coordinate system convention of the SPQEP was defined as the "Volume Grid" coordinate system. Thus, it is possible to perform arbitrary coordinate transforms between the surface determination and the surface export (e. g. necessary within the context of a registration routine). The CT volume data must be available via an open file format (e. g. as .vgi file), in order to be imported into the SPQEP. Those three components (volume data in the .vgi file format, determined surface in the .stl file format and the $4 \times 4$-transformation matrix) are then imported into the SPQEP. Within the .stl file format, three edge points define each triangle. That means that many edge points are defined more than once, depending on the connectivity of triangles. The vertex data and the mesh connectivity matrix are modified in such way, that every unique edge point is

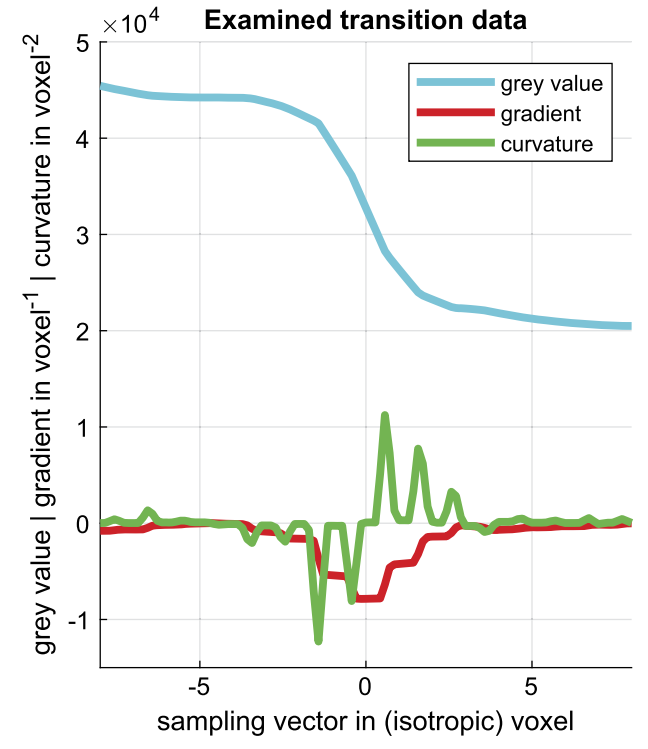

Figure 4: Grey value transition for a real measurement with gradient and curvature.

only defined once within the data structure. Then the vertex normal vectors are calculated, following the convention that the direction of the vector is outward. The triangle mesh then undergoes a clean-up routine, where data consistency problems are addressed and corrected (e. g. incorrect normal vectors, unconnected triangles, and collinear triangle edge points). Knowing the coordinate transformation between surface data and volume data, for each surface point (unique triangle edge point), the grey value profile in the direction of the corresponding vertex normal vector can be constructed (Fig.4). This profile is centred on the surface point location and typically has a user defined (unidirectional) length of 4 to 15 voxels. The x-axis (sampling vector) is counted positively in the direction of the vertex normal vector. The derivatives of the sampled grey values transitions are strongly affected by the fact that the volume data are gridded data (discrete and therefore mathematically unsteady). In order to determine the SPQ for each surface point, the following sources of information are used:

- The form and shape of the sampled grey value transition. This is the main source of information.

- Knowledge of the user defined settings of the CT measurement and the physical interactions during the measurement, e. g. X-ray tube settings, material of the measurement object and positioning of the axis of rotation with respect to the measurement object (in the case of cone beam CT).

- Knowledge of used algorithms and data processing operations and their influence on the measurement re- 
sult, e. g. reconstruction algorithm, user defined settings regarding the surface determination algorithm.

More specifically, each grey value transition can be evaluated for certain criteria, which can then be compared for the complete work piece surface. The local differences of the SPQ represent local changes of the volume data in the proximity of work piece edges and can therefore indicate surface regions with a potentially increased determination uncertainty. The mathematical criteria for the evaluation of grey value transitions include but are not limited to the following examples:

- value and location of the maximum gradient, width of the gradient curve,

- value of the maximum absolute curvature, locations of zero crossings of the curvature,

- $\quad$ symmetry of the transition with respect to the underlying surface point location,

- contrast and monotonicity of the transition,

- grey values at each end of the sampling length with respect to the maximum / minimum grey value,

- local statistical behaviour of the aforementioned criteria.

Within the SPQEP, the grey value profile of each surface point can be examined. The evaluation of the SPQ then takes place by comparing all surface points of the complete dataset using false-colour plots with suited colour maps. The colour map generally changes from green to yellow to red or vice versa, with green indicating the better SPQ by intuitive reasoning (e. g. larger gradient, better symmetry, higher contrast). Usually, expert knowledge is required to choose meaningful SPQ parameters for a given measurement, as well as suitable colour map windowing settings. Often-times a good indicator for choosing an applicable parameter is the evaluation of the selectivity using histogram visualization of all SPQ of a CT measurement. The following section demonstrates some selected applications and use cases for the surface point quality.

\section{Applications and use cases}

This section shows selected scenarios for the application of the SPQ in the context of dimensional metrology. The examples highlight the local classification of various different artefacts and error influences onto the determined surface for various materials including steel, aluminium and polymer. The qualitative separation of work piece surface regions generates potential benefits for subsequent measurement data processing and evaluation steps, such as geometrical registration, geometry element association/regression and generation of improved measurement setups.

An application of the presented method was already demonstrated in the context of metal artefact reduction [19]. The appearance of metal artefacts depends on the direction of X-ray penetration. This fact was used to set up repeated measurements of the same measurement object but with different object orientations during the measurement with respect to the coordinate system of the CT system. The classification of surface regions corrupted by severe artefacts was then used to implement a weighted data fusion routine, using SPQ values [19].

All following measurements were performed using the industrial CT system Zeiss Metrotom 1500 with a fixed source - detector distance of approx. $1377 \mathrm{~mm}$ (the exact value can be determined by a geometric calibration of the CT system).

\subsection{Aluminium hole plate}

The first example features an aluminium hole plate. The measurement settings were chosen as follows: tube voltage $225 \mathrm{kV}$, tube current $511 \mu \mathrm{A}$, integration time $1000 \mathrm{~ms}$, detector amplification 8x, geometrical magnification 1.74x, voxel size $115 \mu \mathrm{m}$, X-ray focal spot size $115 \mu \mathrm{m}$, X-ray pre-filtration $1 \mathrm{~mm} \mathrm{Cu}$, number of projections 2000. Surface determination was performed using VGS with standard settings in the "Advanced" mode. The measurement object was clamped in such way, that the zaxis of the work piece (compare Fig. 5) was aligned parallel with the rotation axis of the rotatory stage of the CT system. For the calculation of the SPQ, the minimum gradient of the grey value transitions (compare also Fig. 4) was evaluated. Including the knowledge of the exact measurement setup, yellow/red coloured surface regions inside the holes can be interpreted as cone beam artefacts. These can be observed at surface regions, which are located inside the "shadow zone" (Fig. 2), which can clearly be observed for the same angular region of all holes of the hole plate. Additionally, the different X-ray penetration lengths can be observed on the surface in the yz plane of the work piece. In this scenario, one quality parameter flags two different types of SPQ degradation mechanisms, which can not be separated intrinsically without additional information. 


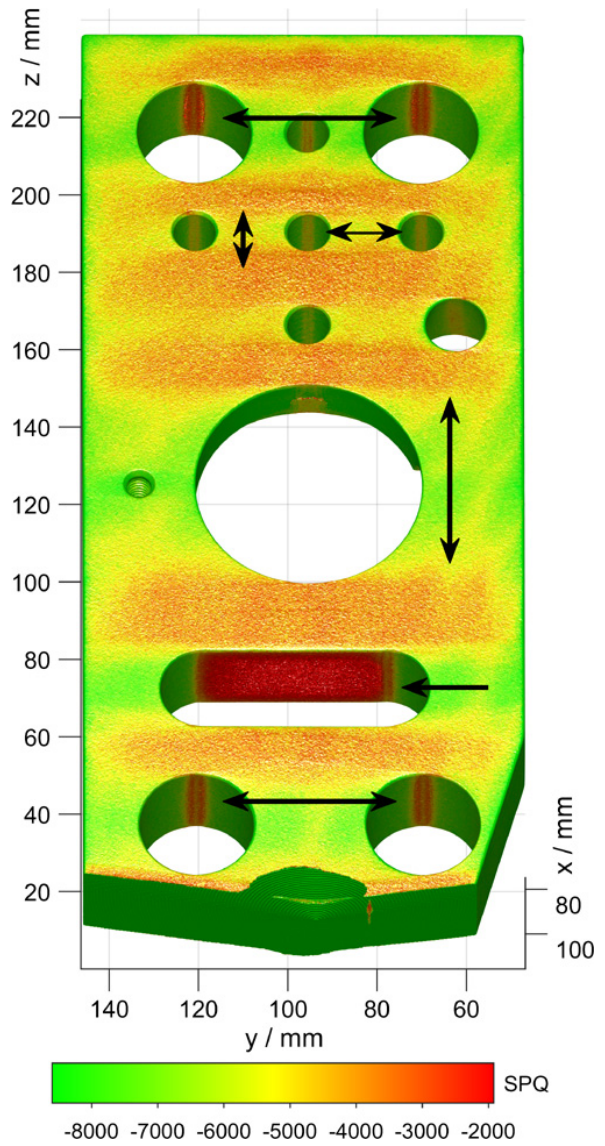

Figure 5: Classification of cone beam artefacts as well as artefacts due to high X-ray penetration lengths using a gradient SPQ criterion. Green coloured regions show surface areas with a high SPQ. Exemplary surfaces areas containing artefacts are highlighted with arrows.

\subsection{Touching steel spheres}

The second example shows severe beam hardening artefacts for the measurement of two touching steel spheres (diameter $14.3 \mathrm{~mm}$ ), which are placed next to each other in X-ray direction. The measurement settings for this measurement were chosen as follows: tube voltage $225 \mathrm{kV}$, tube current $444 \mu \mathrm{A}$, integration time $500 \mathrm{~ms}$, detector amplification 16x, geometrical magnification 10x, voxel size $20 \mu \mathrm{m}$, X-ray focal spot size $100 \mu \mathrm{m}$, no X-ray pre-filtration, number of projections 2000. Surface determination was performed using VGS, using the default settings of the "Advanced" mode. The artefacts locally impede a correct surface determination. The evaluation of the SPQ using a contrast criterion is able to detect the low quality surface regions (Fig. 7). The chosen classification parameter exhibits excellent selectivity properties for this particular use cases. This can be explained by considering the histogram visualization of the SPQ values of the complete

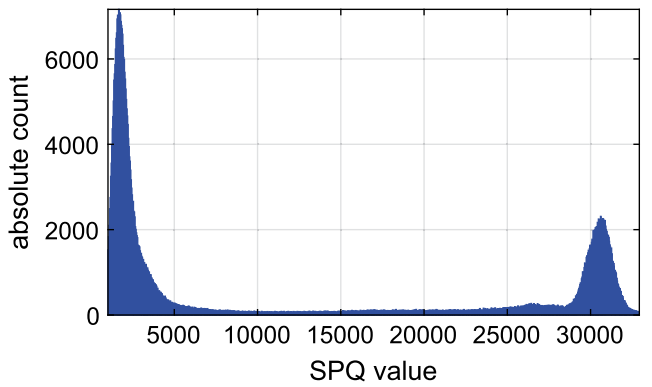

Figure 6: Demonstration of the excellent selectivity of the SPQ criterion for the use case at hand by evaluation of the histogram of the SPQ values of all surface points (larger values indicate a higher surface point quality).

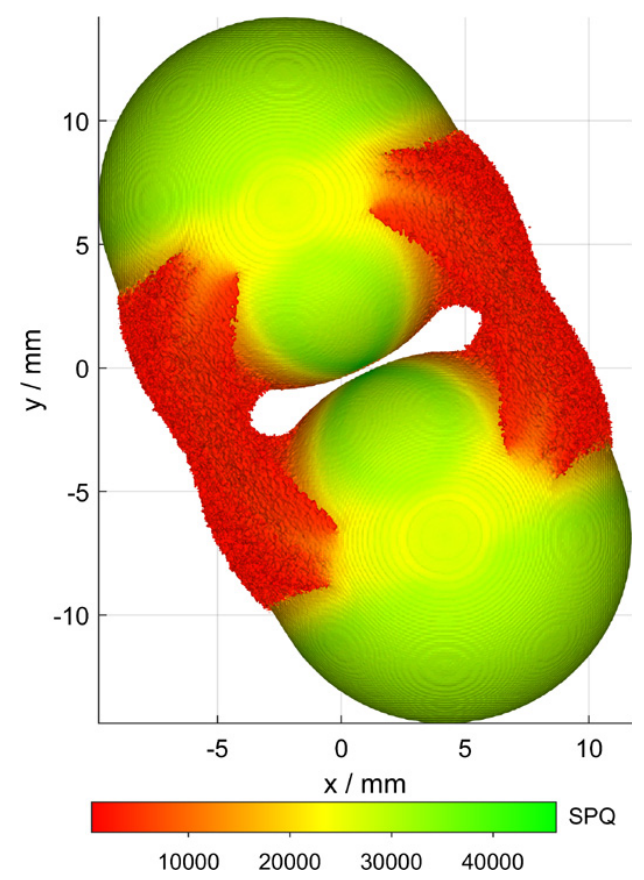

Figure 7: Classification of severe beam hardening artefacts using a SPQ contrast criterion. The ring pattern on the surface are caused by data resampling in VGS in order to reduce the overall number of surface points.

measurement (Fig. 6). The histogram shows two distinct peaks, which makes the selection of an artefact threshold value very easy.

\subsection{Qualitative correlation of the SPQ with the Single Point Scatter}

Until now, the SPQ is just an abstract local quality parameter. To increase the usability in the context of dimensional metrology, a demonstrable correlation of those quality val- 


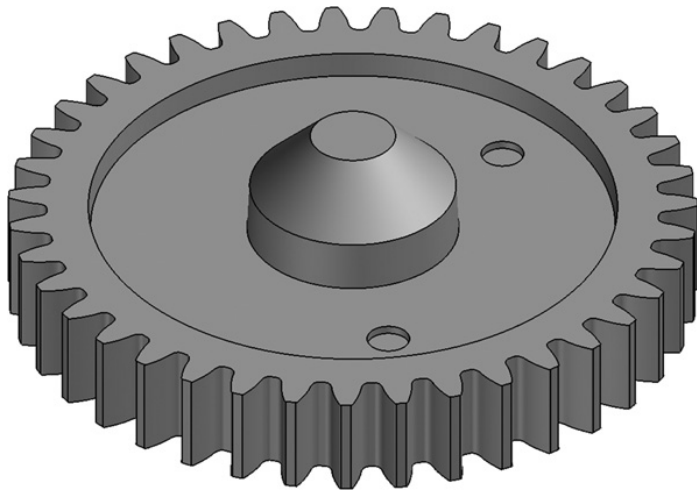

Figure 8: CAD model of the measured polymer gear wheel.

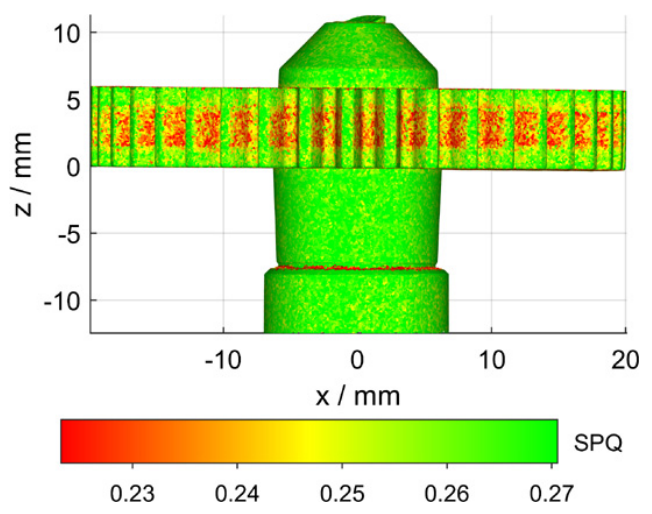

Figure 9: SPQ for the surface of a single measurement evaluating the contrast of the grey value transition.

ues with the observable measurement uncertainty is desired. For this purpose, a measurement series of a polymer gear wheel was recorded (Fig. 9). The middle part of the gear wheel is retracted in relation to the complete width of the gear sprocket, which is an important detail, because it leads to different X-ray penetration lengths in the following example. The measurement settings were chosen as follows: tube voltage $100 \mathrm{kV}$, tube current $250 \mu \mathrm{A}$, integration time $2000 \mathrm{~ms}$, detector amplification 16x, geometrical magnification $8 \mathrm{x}$, voxel size $25 \mu \mathrm{m}$, X-ray focal spot size $25 \mu \mathrm{m}$, no X-ray pre-filtration, number of projections 1500 , number of images averaged for one projection 5 . The rotation axis of the gear wheel was aligned parallel with the rotation axis of the CT system (see also Fig. 9). In former publications [11, 20, 21, 17, 18, 22], the concept of the Single Point Uncertainty was introduced and used to determine the associated measurement uncertainty for arbitrary vertices on a measured surface. For this purpose, repeated measurements are used in conjunction with a reference measurement in order to separate the work piece deviations and the systematic as well as the random mea- surement error for each location on the surface of the measurement object. For this article, the Single Point Scatter (SPSc), which is a measure for the local random measurement error was calculated for the aforementioned measurement series and then compared against the SPQ, using a contrast criterion. If it is possible to demonstrate a correlation between the SPQ and the SPSc, the SPQ could be used to estimate the random measurement error contribution to the measurement uncertainty just from one measurement (remember the calculation of the SPSc requires a complete measurement series consisting of at least 20 single measurements).

Fig. 9 shows the SPQ for a single gear wheel measurement, using a contrast criterion, meaning that surface points with a high contrast grey value transition are rated with a higher quality (green colour). It is observable, that the SPQ decreases for the middle part of the gear sprocket, which is caused by increased X-ray penetration lengths compared to the full width of the gear sprocket. Although the increased penetration lengths do not impede the determination of the surface in this case, the method is sensitive to the small changes in the volume data. Fig. 10 shows the calculated SPSc of the gear wheel sprocket from the aforementioned measurement series. It is observable, that the SPSc also increases with increasing X-ray penetration lengths, which directly indicates a qualitative correlation between the SPQ and the SPSc.

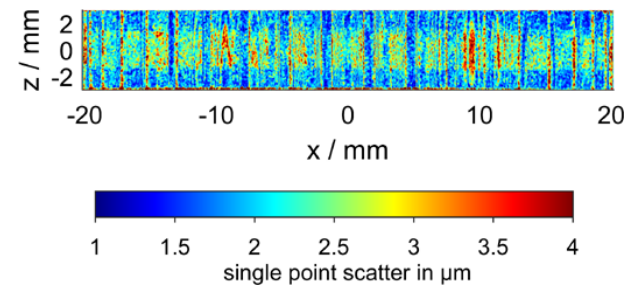

Figure 10: Single Point Scatter (SPSc) of the complete measurement series. Evaluation was intentionally limited to the sprocket in order to reduce computational expenses. The different coordinate system compared to Fig. 9 is caused by the different data processing pipelines.

\section{Currently unsolved problems and challenges}

Currently, one of the main challenges is that SPQ values have an arbitrary scale and can not be compared between different measurements. The reason for that is, that the values are calculated from grey value profiles, which are based on the reconstructed volume. Grey values in volume 
data are typically scaled differently, the meaning is derived from the locality of grey values within the volume. Secondly, volume data exhibit noise, which is even more problematic if the determined SPQ value is based on a derivative of the grey value profile. This is reflected by the fact, that the results could not be confirmed for the measurement series shown in section 4.3 without the image averaging of 5 images (all other settings were exactly identical). Lastly, the judgement of a measurement and the classification of artefacts using the presented method nonetheless requires a deep understanding of the tool and the various interactions and influences affecting the volume data of a CT measurement.

\section{Summary and outlook}

In this article, a method to qualitatively classify surface regions based on the grey value profile along the surface point normal vector was presented. It was shown that the approach can successfully be used to detect surface regions affected by measurement artefacts, such as cone beam artefacts, beam hardening and reduced signal to noise ratio due to increased X-ray penetration lengths. A qualitative correlation between the SPQ and the random measurement error was demonstrated for a polymer gear wheel measurement. Full use of this approach can be made by adjusting the measurement evaluation routines as the presence of a valid surface point quality measure makes it now possible to increase the weight of high quality surface points within the mathematical point cloud processing. Therefore the measurement results achievable with state of the art CT systems can be improved, because the associated measurement uncertainty can directly be reduced. Nonetheless, the main challenges associated with this method are the abstract nature of the determined quality parameters and the requirement of expert knowledge in the field of CT in order to explain the origin of detected artefacts.

Author contributions: "CRediT (Contributor Roles Taxonomy) is high-level taxonomy, including 14 roles, that can be used to represent the roles typically played by contributors to scientific scholarly output. The roles describe each contributor's specific contribution to the scholarly output." [6]

AMM contributed to Conceptualization, Data curation, Formal analysis, Investigation, Methodology, Software, Validation, Visualisation, Writing - original draft and Writing - review \& editing. LB and FW both contributed to Formal Analysis, Visualisation, Writing - original draft and Writing - review \& editing. TH contributed to Conceptualisation, Funding acquisition, Project administration, Supervision and Writing - review \& editing.

Funding: The authors would like to thank the German Research Foundation (DFG) for supporting the research project "FOR 2271 process-oriented tolerance management based on virtual computer-aided engineering tools" under grant number HA 5915/9-2 and for the financial support of the acquisition of the CT system Zeiss Metrotom 1500 through Grant No. 324672600.

Competing interests: For the presented methodology, a patent application with number DE 102015201271 A1 (application date: 26.01.2015 [13]) is pending at the German national patent office. The patent application has also been registered as European application (EP 3195258) and international application (WO 2016042105) on 17.09.2015 [12].

\section{References}

1. ASTM. E1695-95(2013), Standard Test Method for Measurement of Computed Tomography (CT) System Performance, ASTM International, West Conshohocken, PA, 2013.

2. J. F. Barrett and N. Keat. Artifacts in CT: Recognition and avoidance. RadioGraphics, 24 (6): 1679-1691, 2004. ISSN 0271-5333, 1527-1323. 10.1148/rg.246045065.

3. S. Bartolac, R. Clackdoyle, F. Noo, J. Siewerdsen, D. Moseley, and D. Jaffray. A local shift-variant fourier model and experimental validation of circular cone-beam computed tomography artifacts. Medical Physics, 36 (2): 500-512, 2009. 10.1118/1.3062875.

4. T. M. Buzug. Computed Tomography. Springer, Berlin Heidelberg, 2008. 10.1007/978-3-540-39408-2.

5. S. Carmignato, W. Dewulf, and R. Leach, editors. Industrial $X$-Ray Computed Tomography. Springer International Publishing, 2018. 10.1007/978-3-319-59573-3.

6. CASRAI. CRediT: CRT. URL https://www.casrai.org/credit.html.

7. R. Christoph, H. Weise, C. Leinweber, and M. Kachelrieß. Examination of a Method for the Optimization of Scan Parameters for Measuring with Computed Tomography. In 8th Conference on Industrial Computed Tomography, 2018.

8. L. A. Feldkamp, L. C. Davis, and J. W. Kress. Practical cone-beam algorithm. JOSA A, 1 (6): 612-619, 1984. ISSN 1520-8532. 10.1364/JOSAA.1.000612. URL https://www.osapublishing. org/abstract.cfm?uri=josaa-1-6-612.

9. M. Fleßner, A. Müller, E. Helmecke, and T. Hausotte. Automated detection of artefacts for computed tomography in dimensional metrology. In Digital Industrial Radiology and Computed Tomography, 2015. URL http://www.ndt.net/events/DIR2015/ app/content/Paper/35_Flesner.pdf. 
10. M. Fleßner, A. M. Müller, E. Helmecke, and T. Hausotte. Evaluating and visualizing the quality of surface points determined from computed tomography volume data. In PTB open access repository, 2015. 10.7795/810.20150223A.

11. M. Fleßner, A. M. Müller, D. Götz, E. Helmecke, and T. Hausotte. Assessment of the single point uncertainty of dimensional CT measurements. In NDT.net issue Vol. 21 No. 02, 2016. URL https://www.ndt.net/article/ctc2016/papers/ICT2016_paper_ id48.pdf.

12. German Patent and Trade Mark Office (DPMA). register information EP file number: 1577 8622.9 / WO file number: PCT/EP2015/071377. https://register.dpma.de/DPMAregister/ pat/register?AKZ=E157786229, (accessed on 08/15/2019).

13. German Patent and Trade Mark Office (DPMA). register information DE file number : 102015201 271.9. https: //register.dpma.de/DPMAregister/pat/register?AKZ= 1020152012719, (accessed on 08/15/2019).

14. Hanno Krieger, Grundlagen der Strahlungsphysik und des Strahlenschutzes, Springer Spektrum, Vieweg+Teubner Verlag, Wiesbaden, 2012. 10.1007/978-3-8348-2238-3.

15. J. J. Lifton, A. A. Malcolm, and J. W. McBride. A simulation-based study on the influence of beam hardening in $\mathrm{x}$-ray computed tomography for dimensional metrology. Journal of X-Ray Science and Technology, 23 (1): 65-82, 2015. ISSN 0895-3996. 10.3233/XST-140471. URL https://content.iospress.com/ articles/journal-of-x-ray-science-and-technology/xst00471.

16. J. J. Lifton, A. A. Malcolm, and J. W. McBride. On the uncertainty of surface determination in x-ray computed tomography for dimensional metrology. Measurement Science and Technology, 26 (3): 035003, Feb. 2015. ISSN 0957-0233. 10.1088/0957-0233/26/3/035003. URL https://doi.org/10. $1088 \% 2 \mathrm{~F} 0957-0233 \% 2 \mathrm{~F} 26 \% 2 \mathrm{~F} 3 \% 2 \mathrm{~F} 035003$.

17. A. Müller, S. Metzner, and T. Hausotte. Characterization of the single point precision of steel gear wheel measurements using tactile coordinate measurement machines in scanning mode. In AMA Service GmbH, editor, 20. GMA/ITG-Fachtagung Sensoren und Messsysteme 2019, pages 756-762, 2019. ISBN 978-3-9819376-0-2. 10.5162/sensoren2019/P2.18. URL https://www.ama-science.org/proceedings/details/3485.

18. A. Müller, S. Metzner, T. Hausotte, D. Schubert, and D. Drummer. Separation of locally determined work piece deviations and measurement uncertainties for structured-light scanning of customized polymer gear wheels. In AMA Service GmbH, editor, 20. GMA/ITG-Fachtagung Sensoren und Messsysteme 2019, pages 527-534, 2019. ISBN 978-3-9819376-0-2. 10.5162/sensoren2019/6.4.1. URL https://www.ama-science.org/proceedings/details/3467.

19. A. M. Müller and T. Hausotte. Data fusion of surface data sets of X-ray computed tomography measurements using locally determined surface quality values. Journal of Sensors and Sensor Systems, 7: 551-557, 2018. 10.5194/jsss-7-551-2018. URL https://www.j-sens-sens-syst.net/7/551/2018/.

20. A. M. Müller and T. Hausotte. Comparison of different measures for the single point uncertainty in industrial X-ray computed tomography. In 9th Conference on Industrial Computed Tomography. e-Journal of Nondestructive Testing, 2019. URL http://www.ndt.net/iCT2019.
21. A. M. Müller and T. Hausotte. Utilization of single point uncertainties for geometry element regression analysis in dimensional X-ray computed tomography. In 9th Conference on Industrial Computed Tomography. e-Journal of Nondestructive Testing, 2019. URL http://www.ndt.net/iCT2019.

22. A. M. Müller, T. Oberleiter, K. Willner, and T. Hausotte. Implementation of Parameterized Work Piece Deviations and Measurement Uncertainties into Performant Meta-models for an Improved Tolerance Specification. Proceedings of the Design Society: International Conference on Engineering Design, 1 (1): 3501-3510, 2019. 10.1017/dsi.2019.357. URL https://www.cambridge.org/core/services/aop-cambridgecore/content/view/C1C964DDFADF929B3B4454E4D0D4783D/ S2220434219003573a.pdf/implementation_of_ parameterized_work_piece_deviations_and_measurement_ uncertainties_into_performant_metamodels_for_an_ improved_tolerance_specification.pdf.

23. K. Schörner. Development of Methods for Scatter Artifact Correction in Industrial $X$-ray Cone-beam Computed Tomography. phdthesis, Technische Universität München, 2012. URL https://mediatum.ub.tum.de/doc/1097730/ 1097730.pdf.

24. L. A. Shepp and B. F. Logan. The fourier reconstruction of a head section. IEEE Transactions on Nuclear Science, 21 (3): 21-43, June 1974. ISSN 0018-9499. 10.1109/TNS.1974.6499235.

25. H. Tuy. An inversion formula for cone-beam reconstruction. SIAM Journal on Applied Mathematics, 43 (3): 546-552, 1983. ISSN 0036-1399. 10.1137/0143035.

26. C. H. Yan, R. T. Whalen, G. S. Beaupré, S. Y. Yen, and S. Napel. Reconstruction algorithm for polychromatic CT imaging: application to beam hardening correction. IEEE Transactions on Medical Imaging, 19 (1): 1-11, Jan 2000. ISSN 0278-0062. 10.1109/42.832955.

\section{Bionotes}

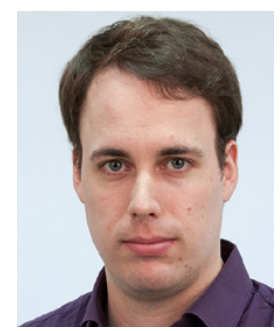

\section{Andreas Michael Müller}

Institute of Manufacturing Metrology (FMT), Friedrich-Alexander-Universität Erlangen-Nürnberg Erlangen, Germany andreas.mueller@fmt.fau.de

Since 2016, Andreas Müller has been a research associate at the Institute of Manufacturing Metrology. His main fields of research are applications of GPU computing and efficient computer vision algorithms for dimensional metrology, industrial X-ray computed tomography and measurement uncertainty analysis. 


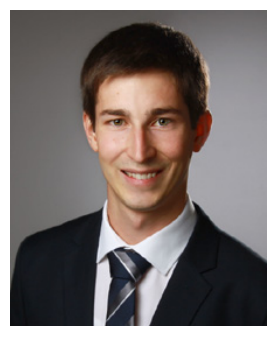

\section{Lorenz Butzhammer}

Institute of Manufacturing Metrology (FMT), Friedrich-Alexander-Universität Erlangen-Nürnberg Erlangen, Germany lorenz.butzhammer@fmt.fau.de

Since 2017, Lorenz Butzhammer has been a research associate at the Institute of Manufacturing Metrology in the X-ray computed tomography group. His main field of research is the optimisation of the scan process to reduce the measurement uncertainty for industrial CT.

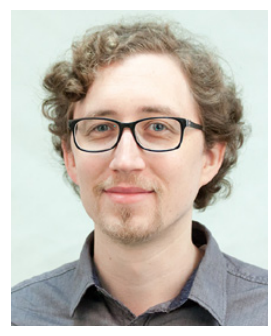

\section{Florian Wohlgemuth}

Institute of Manufacturing Metrology (FMT), Friedrich-Alexander-Universität Erlangen-Nürnberg Erlangen, Germany

florian.wohlgemuth@fmt.fau.de

Since 2017, Florian Wohlgemuth has been a research associate at the Institute of Manufacturing Metrology in the X-ray computed tomography group. His main fields of research are metrological structural resolution, standardisation and realistic simulations of CT measurements.

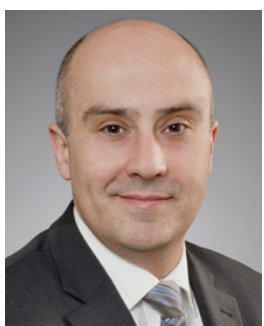

\section{Tino Hausotte}

Institute of Manufacturing Metrology (FMT), Friedrich-Alexander-Universität

Erlangen-Nürnberg Erlangen, Germany tino.hausotte@fmt.fau.de

Tino Hausotte has been professor and head of the Institute of Manufacturing Metrology at the Friedrich-Alexander-University ErlangenNuremberg since 2011. Under his management, the institute specialises on research about the topics X-ray computed tomography, surface and coordinate metrology, micro- and nanometrology, photogrammetry and measurement uncertainty evaluation. 